\title{
Laparoscopic Total Pelvic Exenteration for Locally Recurrent Rectal Cancer
}

\author{
Takashi Akiyoshi, MD, PhD, Toshiya Nagasaki, MD, PhD, and Masashi Ueno, MD, PhD \\ Department of Gastroenterological Surgery, Cancer Institute Hospital, Gastroenterological Center, Japanese Foundation for \\ Cancer Research, Koto-ku, Tokyo, Japan
}

\begin{abstract}
Background. Extended surgery for locally recurrent rectal cancer is technically demanding because of the severe fibrosis around the tumor, which makes it difficult to achieve R0 resection. Although laparoscopic total pelvic exenteration has been carried out in patients with primary rectal cancer, ${ }^{1,2}$ to our knowledge ours is the first report of this laparoscopic procedure for locally recurrent rectal cancer. Methods. A 70-year-old man who underwent laparoscopic low anterior resection for stage II rectal cancer was diagnosed as having two separate local recurrences near the anastomotic site. We decided to perform laparoscopic total pelvic exenteration after neoadjuvant chemoradiotherapy. The branches of the internal iliac vessels were carefully identified and divided. Presacral dissection behind the neorectum was carried out above the anastomotic site. Ligation of the dorsal vein complex was performed under direct visualization, with the patient in the jack-knife position. The perineal defect was reconstructed using a bilateral $\mathrm{V}-\mathrm{Y}$ advancement of the musculocutaneous flaps of the gluteus maximus. An ileal conduit was constructed extracorporeally via an umbilical incision, after placing the patient in the lithotomy position.
\end{abstract}

Electronic supplementary material The online version of this article (doi:10.1245/s10434-015-4473-8) contains supplementary material, which is available to authorized users.

(C) Society of Surgical Oncology 2015

First Received: 6 January 2015;

Published Online: 10 March 2015

T. Akiyoshi, MD, PhD

e-mail: takashi.akiyoshi@jfcr.or.jp
Results. The total operative time was $18 \mathrm{~h}$ and $5 \mathrm{~min}$, with an estimated blood loss of $750 \mathrm{~mL}$. Final pathology showed negative resection margins.

Conclusions. Laparoscopic total pelvic exenteration for locally recurrent rectal cancer is a technically challenging procedure requiring a long operative time. However, as demonstrated by this case, it can provide a very clear view of the operative field, allowing precise dissection, less blood loss, and a smaller abdominal wound.

DISCLOSURE The authors have no potential conflicts of interest.

\section{REFERENCES}

1. Mukai T, Akiyoshi T, Ueno $\mathrm{M}$, et al. Laparoscopic total pelvic exenteration with en bloc lateral lymph node dissection after neoadjuvant chemoradiotherapy for advanced primary rectal cancer. Asian J Endosc Surg. 2013;6:314-17.

2. Patel H, Joseph JV, Amodeo A, Kothari K. Laparoscopic salvage total pelvic exenteration: is it possible post-chemo-radiotherapy? $J$ Minim Access Surg. 2009;5:111-14. 